\title{
HYPERHOLOMORPHIC FUNCTIONS WITH VALUES IN A MODIFIED FORM OF QUATERNIONS
}

\begin{abstract}
We give the definition of hyperholomorphic pseudocomplex functions, i.e., functions with values in a special form of quaternions, and propose the necessary variables, functions, and Dirac operators to describe the Cauchy integral theorem and the generalized Cauchy-Riemman system. We investigate the properties and corollaries corresponding to the Cauchy integral theorem for the pseudo-complex number system discussed in this paper.
\end{abstract}

Key words: hyperholomorphic, quaternion, dirac operator, CauchyRiemman system

2010 Mathematical Subject Classification: 32A30, 30G35, 32W50, 26B05

1. Introduction. The non-commutative four-dimensional space $\mathbb{R}^{4}$ of hypercomplex numbers, which are called quaternions with four real numbers, was studied by Hamilton [5]. Since quaternions involve noncommutative multiplication, quaternions have different algebraic properties compared to the complex number system. In 1935, Fueter [2] defined regular quaternionic functions in $\mathbb{R}^{4}$. Later Deavours [1] and Subdery [12] developed quaternionic analysis, based on complex analysis.

Many formulas in $\mathbb{R}^{4}$ are simpler and more convenient to apply in physics when written in terms of $\mathbb{C}^{2}$. In [11], Nôno represented quaternions in the complex-number form. In [6], Kajiwara et al. gave an integrability condition for any hyperholomorphic function $f_{1}+f_{2} j$ composed of harmonic complex-valued functions $f_{1}$ and $f_{2}$ in a pseudoconvex domain of $\mathbb{C}^{4}$. In [7], [8], Kim et al. presented a ternary representation of real quaternions and also introduced the pseudo-complex number form with the modified basis $\hat{i}$. The regularity of a function defined in $\mathbb{R}^{3}$ relative to the commonly known properties of regular functions was defined.

(C) Petrozavodsk State University, 2020 
Hamilton tried to generalize complex numbers to the form $a+i b+j c$, where $a, b, c \in \mathbb{R}$ and $i^{2}=j^{2}=-1$. However, since the set $\{a+i b+$ $+j c \mid a, b, c \in \mathbb{R}\}$ is not closed under multiplication (which was proved by Kenneth in 1966), this set cannot be generalized as an algebra. Later, Hamilton found a closed multiplication for complex numbers, denoted by $q=i x+j y+k z$, where $i^{2}=j^{2}=k^{2}=i j k=-1$. Some interesting investigations were carried out on the set $\{a+i b+j c \mid a, b, c \in \mathbb{R}\}$. Leutwiler [9] studied the interplay between the solutions $f=u+i v+j w$ of the generalized Cauchy-Riemann system and functions of the reduced quaternionic variable $z=x+i y+j t$. Leutwiler showed that every solution $f$ of that system defined in some neighborhood of the origin admits a series expansion in terms of the elementary polynomial solutions. In [3], [4], Gürlebeck and Sprößig studied quaternion-valued functions that are defined in open subsets of $\mathbb{R}^{n}(n=3,4)$ and are solutions of generalized Cauchy-Riemann or Dirac systems. Their research is related to boundary-value problems and partial differential equations.

This paper recalls the properties resulting from the applications of the defined differential operators and the regularity of modified ternary functions. Using the properties of a modified ternary function, we present integration over the boundary of a domain in the modified ternary numbers. In addition, the present paper presents and verifies the Cauchy integral theorem for modified ternary functions. We also expose corollaries to the Cauchy integral theorem. The paper introduces the definitions of hyperholomorphic functions on the real ternary numbers and represents pseudo-complex numbers as a special form of quaternions, defined as $a+b \hat{i}$. In section 2 , we provide the necessary variables, functions, and operators used in the paper. In section 3, we refer to Naser [10] and Nôno [11] in order to propose Dirac operators and Cauchy integral theorems. And then we introduce the properties and corollaries corresponding to the Cauchy integral theorem for the pseudo-complex number system.

2. Preliminaries. Let $\mathbb{T}$ be the set of all ternary numbers:

$$
\mathbb{T}=\left\{z \mid z=x_{0}+x_{1} e_{1}+x_{2} e_{2}, \quad x_{0}, x_{1}, x_{2} \in \mathbb{R}\right\},
$$

where $e_{1}^{2}=e_{2}^{2}=-1$ and $e_{1} e_{2}=\sqrt{-1}$. An element $z$ of $\mathbb{T}$ can be written as

$$
\begin{aligned}
z & =x_{0}+x_{1} e_{1}+x_{2} e_{2}= \\
& =x_{0}+\frac{a e_{1}+b e_{2}}{\sqrt{a^{2}+b^{2}}}\left(\frac{\sqrt{a^{2}+b^{2}}}{a e_{1}+b e_{2}} x_{1} e_{1}+\frac{\sqrt{a^{2}+b^{2}}}{a e_{1}+b e_{2}} x_{2} e_{2}\right)=
\end{aligned}
$$




$$
=x_{0}+\frac{a e_{1}+b e_{2}}{\sqrt{a^{2}+b^{2}}}\left(\frac{a x_{1}+b x_{2}}{\sqrt{a^{2}+b^{2}}}+\frac{b x_{1}-a x_{2}}{\sqrt{a^{2}+b^{2}}} e_{1} e_{2}\right),
$$

where $a$ and $b$ are real non-zero numbers. Let $\hat{i}$ be the modified basis in $\mathbb{T}$, denoted by

$$
\hat{i}=\frac{a e_{1}+b e_{2}}{\sqrt{a^{2}+b^{2}}} \quad \text { and } \quad \hat{i}^{2}=-1 .
$$

Then, an element $z$ of $\mathbb{T}$ can also be written as

$$
z=x_{0}+\hat{i} z_{0}
$$

such a number is called a pseudo-complex number; here

$$
z_{0}=\frac{a x_{1}+b x_{2}}{\sqrt{a^{2}+b^{2}}}+\frac{b x_{1}-a x_{2}}{\sqrt{a^{2}+b^{2}}} e_{1} e_{2} .
$$

The set of pseudo-complex numbers, denoted by $\mathbb{P}$, is isomorphic to $\mathbb{R} \times \mathbb{C}$; that is, $\mathbb{P} \cong \mathbb{R} \times \mathbb{C}$. The addition and multiplication for pseudo-complex numbers are given by

$$
\begin{aligned}
z \pm w & =\left(x_{0}+\hat{i} z_{0}\right) \pm\left(y_{0}+\hat{i} w_{0}\right)= \\
& =\left(x_{0} \pm y_{0}\right)+\hat{i}\left(z_{0} \pm w_{0}\right)
\end{aligned}
$$

and

$$
\begin{aligned}
z w & =\left(x_{0}+\hat{i} z_{0}\right)\left(y_{0}+\hat{i} w_{0}\right)= \\
& =\left(x_{0} y_{0}-\bar{z}_{0} w_{0}\right)+\hat{i}\left(x_{0} w_{0}+z_{0} y_{0}\right),
\end{aligned}
$$

respectively. From the multiplication over $\mathbb{P}$, we can obtain $z_{0} \hat{i}=\hat{i} \bar{z}_{0}$. Hence, the multiplication over $\mathbb{P}$ is closed and associative but not commutative.

Let $\bar{z}$ be the conjugate of $z$, denoted by $\bar{z}=x_{0}-\hat{i} z_{0}$ with $z \bar{z}=\bar{z} z$. Also, the norm $|\cdot|$ is written by

$$
|z|:=\sqrt{z \bar{z}}=\sqrt{x_{0}^{2}+\bar{z}_{0} z_{0}}=\sqrt{x_{0}^{2}+x_{1}^{2}+x_{2}^{2}} .
$$

The inverse element $z^{-1}$ of $\mathbb{P}$ is denoted by

$$
z^{-1}=\frac{\bar{z}}{|z|^{2}} .
$$


Now, consider the definition of hyperholomorphy for pseudo-complex functions. First, the differential operators are given by

$$
D=\frac{\partial}{\partial x_{0}}-\hat{i} \frac{\partial}{\partial \bar{z}_{0}}=\frac{\partial}{\partial x_{0}}-e_{1} \frac{\partial}{\partial x_{1}}-e_{2} \frac{\partial}{\partial x_{2}}
$$

and

$$
\bar{D}=\frac{\partial}{\partial x_{0}}+\hat{i} \frac{\partial}{\partial \bar{z}_{0}}=\frac{\partial}{\partial x_{0}}+e_{1} \frac{\partial}{\partial x_{1}}+e_{2} \frac{\partial}{\partial x_{2}},
$$

where

$$
\begin{aligned}
\frac{\partial}{\partial z_{0}}=\frac{1}{2} \frac{\partial}{\partial x_{1}}( & \left.\frac{a}{\sqrt{a^{2}+b^{2}}}-\frac{b}{\sqrt{a^{2}+b^{2}}} e_{1} e_{2}\right)+ \\
& +\frac{\partial}{\partial x_{2}}\left(\frac{b}{\sqrt{a^{2}+b^{2}}}+\frac{a}{\sqrt{a^{2}+b^{2}}} e_{1} e_{2}\right)= \\
= & \frac{1}{2}\left(\frac{a}{\sqrt{a^{2}+b^{2}}} \frac{\partial}{\partial x_{1}}+\frac{b}{\sqrt{a^{2}+b^{2}}} \frac{\partial}{\partial x_{2}}\right)- \\
& -\left(\frac{b}{\sqrt{a^{2}+b^{2}}} \frac{\partial}{\partial x_{1}}-\frac{a}{\sqrt{a^{2}+b^{2}}} \frac{\partial}{\partial x_{2}}\right) e_{1} e_{2}
\end{aligned}
$$

and

$$
\begin{aligned}
\frac{\partial}{\partial \bar{z}_{0}}=\frac{1}{2} \frac{\partial}{\partial x_{1}}( & \left.\frac{a}{\sqrt{a^{2}+b^{2}}}+\frac{b}{\sqrt{a^{2}+b^{2}}} e_{1} e_{2}\right)+ \\
& +\frac{\partial}{\partial x_{2}}\left(\frac{b}{\sqrt{a^{2}+b^{2}}}-\frac{a}{\sqrt{a^{2}+b^{2}}} e_{1} e_{2}\right)= \\
= & \frac{1}{2}\left(\frac{a}{\sqrt{a^{2}+b^{2}}} \frac{\partial}{\partial x_{1}}+\frac{b}{\sqrt{a^{2}+b^{2}}} \frac{\partial}{\partial x_{2}}\right)+ \\
& +\left(\frac{b}{\sqrt{a^{2}+b^{2}}} \frac{\partial}{\partial x_{1}}-\frac{a}{\sqrt{a^{2}+b^{2}}} \frac{\partial}{\partial x_{2}}\right) e_{1} e_{2} .
\end{aligned}
$$

Then, the Laplacian operator is given by

$$
\begin{aligned}
\Delta & :=D \bar{D}=\bar{D} D=\left(\frac{\partial}{\partial x_{0}}-\hat{i} \frac{\partial}{\partial \bar{z}_{0}}\right)\left(\frac{\partial}{\partial x_{0}}+\hat{i} \frac{\partial}{\partial \bar{z}_{0}}\right)= \\
& =\frac{\partial^{2}}{\partial x_{0}^{2}}+\frac{\partial^{2}}{\partial z_{0} \partial \bar{z}_{0}}=\frac{\partial^{2}}{\partial x_{0}^{2}}+\frac{\partial^{2}}{\partial x_{1}^{2}}+\frac{\partial^{2}}{\partial x_{2}^{2}} .
\end{aligned}
$$


3. Properties of hyperholomorphic functions. Let $\Omega$ be a domain in $\mathbb{R}^{3}$. Consider a function $f$ defined on $\Omega$ and with values in $\mathbb{P i}$ such that $f: \Omega \rightarrow \mathbb{P}$ is defined by

$$
f=u_{0}+u_{1} e_{1}+u_{2} e_{2}=u_{0}+\hat{i} f_{0} .
$$

That is, $f$ satisfies

$$
z=\left(x_{0}, x_{1}, x_{2}\right) \in \Omega \mapsto f(z)=u_{0}\left(x_{0}, x_{1}, x_{2}\right)+\hat{i} f_{0}\left(x_{0}, x_{1}, x_{2}\right) \in \mathbb{P},
$$

where $u_{r}(r=0,1,2)$ are real-valued functions and

$$
f_{0}=\frac{a u_{1}+b u_{2}}{\sqrt{a^{2}+b^{2}}}+\frac{b u_{1}-a u_{2}}{\sqrt{a^{2}+b^{2}}} e_{1} e_{2}
$$

is a complex-valued function. The function $f$ is called a pseudo-complex function. Let the differential operators defined in Section 2 be applied to a function $f: \Omega \rightarrow \mathbb{P}$. Then we have the following equalities:

$$
D f=\left(\frac{\partial}{\partial x_{0}}-\hat{i} \frac{\partial}{\partial \bar{z}_{0}}\right)\left(u_{0}+\hat{i} f_{0}\right)=\left(\frac{\partial u_{0}}{\partial x_{0}}+\frac{\partial f_{0}}{\partial z_{0}}\right)+\hat{i}\left(\frac{\partial f_{0}}{\partial x_{0}}-\frac{\partial u_{0}}{\partial \bar{z}_{0}}\right)
$$

and

$$
\bar{D} f=\left(\frac{\partial}{\partial x_{0}}+\hat{i} \frac{\partial}{\partial \bar{z}_{0}}\right)\left(u_{0}+\hat{i} f_{0}\right)=\left(\frac{\partial u_{0}}{\partial x_{0}}-\frac{\partial f_{0}}{\partial z_{0}}\right)+\hat{i}\left(\frac{\partial f_{0}}{\partial x_{0}}+\frac{\partial u_{0}}{\partial \bar{z}_{0}}\right) .
$$

Since the set $\mathbb{P}$ has non-commutative multiplication, we also apply operators to the function $f$ from the right. We have

$$
f D=\left(u_{0}+\hat{i} f_{0}\right)\left(\frac{\partial}{\partial x_{0}}-\hat{i} \frac{\partial}{\partial \bar{z}_{0}}\right)=\left(\frac{\partial u_{0}}{\partial x_{0}}+\frac{\partial \overline{f_{0}}}{\partial \bar{z}_{0}}\right)+\hat{i}\left(\frac{\partial f_{0}}{\partial x_{0}}-\frac{\partial u_{0}}{\partial \bar{z}_{0}}\right)
$$

and

$$
f \bar{D}=\left(u_{0}+\hat{i} f_{0}\right)\left(\frac{\partial}{\partial x_{0}}+\hat{i} \frac{\partial}{\partial \bar{z}_{0}}\right)=\left(\frac{\partial u_{0}}{\partial x_{0}}-\frac{\partial \overline{f_{0}}}{\partial \bar{z}_{0}}\right)+\hat{i}\left(\frac{\partial f_{0}}{\partial x_{0}}+\frac{\partial u_{0}}{\partial \bar{z}_{0}}\right)
$$

Hence, the equality $\bar{D} f=0$ implies that $f$ satisfies the following equations:

$$
\frac{\partial u_{0}}{\partial x_{0}}=\frac{\partial f_{0}}{\partial z_{0}} \quad \text { and } \quad \frac{\partial f_{0}}{\partial x_{0}}=-\frac{\partial u_{0}}{\partial \bar{z}_{0}}
$$


called the (left-)pseudo-complex Cauchy-Riemann equations. Similarly, if $f$ satisfies $f \bar{D}=0$ then we obain the equations:

$$
\frac{\partial u_{0}}{\partial x_{0}}=\frac{\partial \overline{f_{0}}}{\partial \bar{z}_{0}} \quad \text { and } \quad \frac{\partial f_{0}}{\partial x_{0}}=-\frac{\partial u_{0}}{\partial \bar{z}_{0}}
$$

called the (right-)pseudo-complex Cauchy-Riemann equations. Basing on the definition of the Laplacian, we also obtain

$$
\begin{aligned}
\Delta f & =(D \bar{D}) f=\left(\frac{\partial^{2}}{\partial x_{0}^{2}}+\frac{\partial^{2}}{\partial z_{0} \partial \bar{z}_{0}}\right)\left(u_{0}+\hat{i} f_{0}\right)= \\
& =\left(\frac{\partial^{2} u_{0}}{\partial x_{0}^{2}}+\frac{\partial^{2} u_{0}}{\partial z_{0} \partial \bar{z}_{0}}\right)+\hat{i}\left(\frac{\partial^{2} f_{0}}{\partial x_{0}^{2}}+\frac{\partial^{2} f_{0}}{\partial \bar{z}_{0} \partial z_{0}}\right)
\end{aligned}
$$

Since multiplication over $\mathbb{P}$ is associative,

$$
(D \bar{D}) f=D(\bar{D} f)
$$

Therefore,

$$
\begin{aligned}
D(\bar{D} f)= & \left(\frac{\partial}{\partial x_{0}}-\hat{i} \frac{\partial}{\partial \bar{z}_{0}}\right)\left\{\left(\frac{\partial u_{0}}{\partial x_{0}}-\frac{\partial f_{0}}{\partial z_{0}}\right)+\hat{i}\left(\frac{\partial f_{0}}{\partial x_{0}}+\frac{\partial u_{0}}{\partial \bar{z}_{0}}\right)\right\}= \\
= & \frac{\partial^{2} u_{0}}{\partial x_{0}^{2}}-\frac{\partial^{2} f_{0}}{\partial x_{0} \partial z_{0}}+\hat{i}\left(\frac{\partial^{2} f_{0}}{\partial x_{0}^{2}}+\frac{\partial^{2} u_{0}}{\partial x_{0} \partial \bar{z}_{0}}\right)- \\
& -\hat{i}\left(\frac{\partial^{2} u_{0}}{\partial \bar{z}_{0} \partial x_{0}}-\frac{\partial^{2} f_{0}}{\partial \bar{z}_{0} \partial z_{0}}\right)+\left(\frac{\partial^{2} f_{0}}{\partial z_{0} \partial x_{0}}+\frac{\partial^{2} u_{0}}{\partial z_{0} \partial \bar{z}_{0}}\right)= \\
= & \left(\frac{\partial^{2} u_{0}}{\partial x_{0}^{2}}+\frac{\partial^{2} u_{0}}{\partial z_{0} \partial \bar{z}_{0}}\right)+\hat{i}\left(\frac{\partial^{2} f_{0}}{\partial x_{0}^{2}}+\frac{\partial^{2} f_{0}}{\partial \bar{z}_{0} \partial z_{0}}\right) .
\end{aligned}
$$

Definition 1. Let $\Omega$ be an open set in $\mathbb{R}^{3}$. A function $f: \Omega \rightarrow \mathbb{P}$,

$$
f(z)=u_{0}\left(x_{0}, x_{1}, x_{2}\right)+\hat{i} f_{0}\left(x_{0}, x_{1}, x_{2}\right),
$$

is said to be left-hyperholomorphic on $\Omega$ if $f$ satisfies the following two conditions:

1) $u_{0}$ is a real-analytic function and $f_{0}$ is a holomorphic function,

2) $f$ satisfies the equation $\bar{D} f=0$ on $\Omega$.

Involving the non-commutativity of multiplication, comparing (1) and (2), we also give the following 
Definition 2. Let $\Omega$ be an open set in $\mathbb{R}^{3}$. A function $f: \Omega \rightarrow \mathbb{P}$,

$$
f(z)=u_{0}\left(x_{0}, x_{1}, x_{2}\right)+\hat{i} f_{0}\left(x_{0}, x_{1}, x_{2}\right)
$$

is said to be right hyperholomorphic on $\Omega$ if $f$ satisfies the following two conditions:

1) $u_{0}$ is a real-analytic function and $f_{0}$ is a holomorphic function.

2) $f$ satisfies the equation $f \bar{D}=0$ on $\Omega$,

Since a right hyperholomorphic function is dealt with in a similar manner as a left hyperholomorphic function, we only consider left hyperholomorphic functions and simply call them hyperholomorphic.

Proposition 1 . Let $\Omega$ be an open set in $\mathbb{R}^{3}$ and $f$ be a hyperholomorphic function on $\Omega$. Then

$$
D f=f^{\prime}=\frac{\partial f}{\partial x_{0}}=-\hat{i} \frac{\partial f}{\partial \overline{z_{0}}}
$$

Proof. Since $f$ is a hyperholomorphic function on $\Omega$, (1) yields

$$
D f=\left(\frac{\partial u_{0}}{\partial x_{0}}+\frac{\partial f_{0}}{\partial z_{0}}\right)+\hat{i}\left(\frac{\partial f_{0}}{\partial x_{0}}-\frac{\partial u_{0}}{\partial \bar{z}_{0}}\right)=\frac{\partial u_{0}}{\partial x_{0}}+\hat{i} \frac{\partial f_{0}}{\partial x_{0}}=\frac{\partial}{\partial x_{0}} f .
$$

Moreover, by (1), for $D f$ we also have

$D f=\frac{\partial f_{0}}{\partial z_{0}}-\hat{i} \frac{\partial u_{0}}{\partial \bar{z}_{0}}=-\hat{i}^{2} \frac{\partial f_{0}}{\partial z_{0}}-\hat{i} \frac{\partial u_{0}}{\partial \bar{z}_{0}}=-\hat{i}\left(\frac{\partial}{\partial \bar{z}_{0}} \hat{i} f_{0}+\frac{\partial}{\partial \bar{z}_{0}} u_{0}\right)=-\hat{i} \frac{\partial}{\partial \bar{z}_{0}} f$.

Let us now consider the properties of hyperholomorphic functions in pseudo-complex numbers.

Proposition 2. Let $\Omega$ be an open set in $\mathbb{R}^{3}$ and $f$ and $g$ be hyperholomorphic functions on $\Omega$. Then

1) $\alpha f$ is hyperholomorphic on $\Omega$ if $\alpha$ is any real constant,

2) $f \alpha$ is hyperholomorphic on $\Omega$ if $\alpha$ is any ternary constant,

3) $f \pm g$ is hyperholomorphic on $\Omega$.

Proof. The condition that $f$ and $g$ are both hyperholomorphic functions means that they satisfy (1). For proving items 1) - 3), it suffices to satisfy the second condition of Definition 1. 
1) When $\alpha$ is any real constant, it is obvious that $\bar{D}(\alpha f)=0$.

2) Let $\alpha$ be a pseudo-complex constant, $\alpha=a_{0}+\hat{i} \alpha_{0}$, where $a_{0}$ is real and

$$
\alpha_{0}=\frac{c_{1} a_{1}+c_{2} a_{2}}{\sqrt{c_{1}^{2}+c_{2}^{2}}}+\frac{c_{2} a_{1}-c_{1} a_{2}}{\sqrt{c_{1}^{2}+c_{2}^{2}}} e_{1} e_{2}
$$

with $c_{r}$ and $a_{r}(r=1,2)$ being real numbers. By (1), we infer

$$
\begin{aligned}
& \bar{D}(f \alpha)=\left(\frac{\partial}{\partial x_{0}}+\right.\left.\hat{i} \frac{\partial}{\partial \bar{z}_{0}}\right)\left\{\left(u_{0} a_{0}-\overline{f_{0}} \alpha_{0}\right)+\hat{i}\left(u_{0} \alpha_{0}+f_{0} a_{0}\right)\right\}= \\
&=\left(\frac{\partial u_{0}}{\partial x_{0}} a_{0}-\frac{\partial \overline{f_{0}}}{\partial x_{0}} \alpha_{0}-\frac{\partial u_{0}}{\partial z_{0}} \alpha_{0}-\frac{\partial f_{0}}{\partial z_{0}} a_{0}\right)+ \\
& \quad+\hat{i}\left(\frac{\partial u_{0}}{\partial x_{0}} \alpha_{0}+\frac{\partial f_{0}}{\partial x_{0}} a_{0}+\frac{\partial u_{0}}{\partial \bar{z}_{0}} a_{0}-\frac{\partial \overline{f_{0}}}{\partial \bar{z}_{0}} \alpha_{0}\right)=0 .
\end{aligned}
$$

3) Since $f$ and $g$ are hyperholomorphic functions on $\Omega$, we have

$$
\begin{aligned}
\bar{D}(f \pm g)=\left(\frac{\partial}{\partial x_{0}}+\hat{i} \frac{\partial}{\partial \bar{z}_{0}}\right)\left\{\left(u_{0} \pm v_{0}\right)+\hat{i}\left(f_{0} \pm g_{0}\right)\right\}= \\
=\left(\frac{\partial u_{0}}{\partial x_{0}} \pm \frac{\partial v_{0}}{\partial x_{0}}-\frac{\partial f_{0}}{\partial z_{0}} \mp \frac{\partial g_{0}}{\partial z_{0}}\right)+ \\
\quad+\hat{i}\left(\frac{\partial u_{0}}{\partial \bar{z}_{0}} \pm \frac{\partial v_{0}}{\partial \bar{z}_{0}}+\frac{\partial f_{0}}{\partial x_{0}} a_{0} \pm \frac{\partial g_{0}}{\partial x_{0}}\right)=0 .
\end{aligned}
$$

Example. Let $\Omega$ be an open set in $\mathbb{R}^{3}$ and $f$ and $g$ be hyperholomorphic functions on $\Omega$. Then $f g$ is not always hyperholomorphic on $\Omega$. Since $f$ and $g$ are hyperholomorphic functions on $\Omega$, we obtain

$$
\begin{aligned}
\bar{D}(f g)=\left(\frac{\partial}{\partial x_{0}}+\hat{i} \frac{\partial}{\partial \bar{z}_{0}}\right)\left\{\left(u_{0} v_{0}-\overline{f_{0}} g_{0}\right)+\hat{i}\left(u_{0} g_{0}+f_{0} v_{0}\right)\right\}= \\
=\left(\frac{\partial u_{0}}{\partial x_{0}}-\frac{\partial f_{0}}{\partial z_{0}}\right) v_{0}+u_{0}\left(\frac{\partial v_{0}}{\partial x_{0}}-\frac{\partial g_{0}}{\partial z_{0}}\right)- \\
-\left(\frac{\partial \overline{f_{0}}}{\partial x_{0}}+\frac{\partial u_{0}}{\partial z_{0}}\right) g_{0}-\left(\overline{f_{0}} \frac{\partial g_{0}}{\partial x_{0}}+f_{0} \frac{\partial v_{0}}{\partial z_{0}}\right)+ \\
+\hat{i}\left\{\left(\frac{\partial u_{0}}{\partial x_{0}}-\frac{\partial \overline{f_{0}}}{\partial \bar{z}_{0}}\right) g_{0}+u_{0}\left(\frac{\partial g_{0}}{\partial x_{0}}+\frac{\partial v_{0}}{\partial \bar{z}_{0}}\right)\right\}+
\end{aligned}
$$




$$
\begin{aligned}
+ & \hat{i}\left\{\left(\frac{\partial f_{0}}{\partial x_{0}}+\frac{\partial u_{0}}{\partial \bar{z}_{0}}\right) v_{0}+\left(f_{0} \frac{\partial v_{0}}{\partial x_{0}}-\overline{f_{0}} \frac{\partial g_{0}}{\partial \bar{z}_{0}}\right)\right\}= \\
& =-\overline{f_{0}} \frac{\partial g_{0}}{\partial x_{0}}-f_{0} \frac{\partial v_{0}}{\partial z_{0}}+\hat{i} f_{0} \frac{\partial v_{0}}{\partial x_{0}}-\hat{i} \overline{f_{0}} \frac{\partial g_{0}}{\partial \bar{z}_{0}}= \\
& =\overline{f_{0}} \frac{\partial v_{0}}{\partial \bar{z}_{0}}-f_{0} \frac{\partial v_{0}}{\partial z_{0}}+\hat{i} f_{0} \frac{\partial g_{0}}{\partial z_{0}}-\hat{i} \overline{f_{0}} \frac{\partial g_{0}}{\partial \bar{z}_{0}}=\overline{f_{0}} \frac{\partial g}{\partial \bar{z}_{0}}-f_{0} \frac{\partial g}{\partial z_{0}} .
\end{aligned}
$$

If $\bar{f}_{0} \frac{\partial g}{\partial \bar{z}_{0}}-f_{0} \frac{\partial g}{\partial z_{0}}=0$ then the function $f g$ is hyperholomorphic on $\Omega$. For example, if $f$ is a real-valued function then $f g$ is hyperholomorphic on $\Omega$. However, when $\overline{f_{0}} \frac{\partial g}{\partial \bar{z}_{0}} \neq f_{0} \frac{\partial g}{\partial z_{0}}, f g$ is not hyperholomorphic on $\Omega$.

Put

$$
\omega=d z_{0} \wedge d \bar{z}_{0}-\hat{i} d x_{0} \wedge d z_{0}
$$

Theorem 1. Let $\Omega$ be a domain in $\mathbb{R}^{3}$ and $U$ be any domain in $\Omega$ with smooth boundary $b U$ such that $\bar{U} \subset \Omega$. If $f$ is a hyperholomorphic on $\Omega$ then

$$
\int_{b U} \omega f=0 .
$$

Proof. We have

$$
\begin{aligned}
\omega f & =\left(d z_{0} \wedge d \bar{z}_{0}-\hat{i} d x_{0} \wedge d z_{0}\right)\left(u_{0}+\hat{i} f_{0}\right)= \\
& =\left(u_{0} d z_{0} \wedge d \bar{z}_{0}+f_{0} d x_{0} \wedge d \bar{z}_{0}\right)+\hat{i}\left(f_{0} d \bar{z}_{0} \wedge d z_{0}-u_{0} d x_{0} \wedge d z_{0}\right) .
\end{aligned}
$$

Let $\partial$ and $\bar{\partial}$ be the following operators:

$$
\partial=\frac{1}{2} \frac{\partial}{\partial x_{0}} d x_{0}+\frac{\partial}{\partial z_{0}} d z_{0} \quad \text { and } \quad \bar{\partial}=\frac{1}{2} \frac{\partial}{\partial x_{0}} d x_{0}+\frac{\partial}{\partial \bar{z}_{0}} d \bar{z}_{0} .
$$

Then

$$
\begin{aligned}
& d(\omega f)=(\partial+\bar{\partial})(\omega f)=\left(\frac{\partial}{\partial x_{0}} d x_{0}+\frac{\partial}{\partial z_{0}} d z_{0}+\frac{\partial}{\partial \bar{z}_{0}} d \bar{z}_{0}\right)(\omega f)= \\
& \quad=\left(\frac{\partial u_{0}}{\partial x_{0}}-\frac{\partial f_{0}}{\partial z_{0}}\right) d x_{0} \wedge d z_{0} \wedge d \bar{z}_{0}+\hat{i}\left(\frac{\partial f_{0}}{\partial x_{0}}+\frac{\partial u_{0}}{\partial \bar{z}_{0}}\right) d x_{0} \wedge d z_{0} \wedge d \bar{z}_{0}=0
\end{aligned}
$$

in $U$. It now suffices to apply the Stokes theorem.

Theorem 2. Let $\Omega$ be an open set in $\mathbb{R}^{3}$. If $f$ is hyperholomorphic on $\Omega$ then $u_{0}$ and $f_{0}$ are harmonic functions on $\Omega$. Moreover, $f$ is harmonic on $\Omega$. 
Proof. It suffices to show that $\Delta u_{0}=0$ and $\Delta f_{0}=0$. Indeed, we have

$$
\begin{gathered}
\Delta u_{0}=(D \bar{D}) u_{0}=\frac{\partial^{2} u_{0}}{\partial x_{0}^{2}}+\frac{\partial^{2} u_{0}}{\partial z_{0} \partial \bar{z}_{0}}=\frac{\partial}{\partial x_{0}} \frac{\partial f_{0}}{\partial z_{0}}-\frac{\partial}{\partial z_{0}} \frac{\partial f_{0}}{\partial x_{0}}=0 \\
\Delta f_{0}=(D \bar{D}) f_{0}=\frac{\partial^{2} f_{0}}{\partial x_{0}^{2}}+\frac{\partial^{2} f_{0}}{\partial z_{0} \partial \bar{z}_{0}}=-\frac{\partial}{\partial x_{0}} \frac{\partial u_{0}}{\partial \bar{z}_{0}}+\frac{\partial}{\partial \bar{z}_{0}} \frac{\partial u_{0}}{\partial x_{0}}=0 .
\end{gathered}
$$

By these equalities and (1), we can obtain the equality $\Delta f=0$ as follows:

$$
\begin{aligned}
& \Delta f=(D \bar{D}) f=\left(\frac{\partial^{2} u_{0}}{\partial x_{0}^{2}}-\frac{\partial^{2} f_{0}}{\partial x_{0} \partial z_{0}}+\frac{\partial^{2} f_{0}}{\partial z_{0} \partial x_{0}}+\frac{\partial^{2} u_{0}}{\partial z_{0} \partial \bar{z}_{0}}\right)+ \\
&+\hat{i}\left(\frac{\partial^{2} f_{0}}{\partial x_{0}^{2}}+\frac{\partial^{2} u_{0}}{\partial x_{0} \partial \bar{z}_{0}}-\frac{\partial^{2} u_{0}}{\partial \bar{z}_{0} \partial x_{0}}+\frac{\partial^{2} f_{0}}{\partial \bar{z}_{0} \partial z_{0}}\right)= \\
&=\frac{\partial}{\partial x_{0}}\left(\frac{\partial u_{0}}{\partial x_{0}}-\frac{\partial f_{0}}{\partial z_{0}}\right)+\frac{\partial}{\partial z_{0}}\left(\frac{\partial f_{0}}{\partial x_{0}}+\frac{\partial u_{0}}{\partial \bar{z}_{0}}\right)+ \\
& \quad+\hat{i} \frac{\partial}{\partial x_{0}}\left(\frac{\partial f_{0}}{\partial x_{0}}+\frac{\partial u_{0}}{\partial \bar{z}_{0}}\right)+\hat{i} \frac{\partial}{\partial \bar{z}_{0}}\left(-\frac{\partial u_{0}}{\partial x_{0}}+\frac{\partial f_{0}}{\partial z_{0}}\right)=0 .
\end{aligned}
$$

Thus, $f$ is a harmonic function on $\Omega$.

Consider the following example related to the statement presented in Theorem 2.

Example. Let $u_{0}$ be a real-valued harmonic function such that

$$
u_{0}(z)=\frac{x_{0}}{|z|^{4}}
$$

in a domain $D \subset \mathbb{P}$. Then the hyper-conjugate harmonic function $f_{0}$ of $u_{0}$ can be found in $D$ as

$$
f_{0}=-\frac{z_{0}}{|z|^{4}}
$$

Moreover, $u_{0}+\hat{i} f_{0}$ is hyperholomorphic in $D$.

The following theorem is the Cauchy integral formula for a hyperholomorphic function in $\mathbb{P}$.

Theorem 3. Let $\Omega$ be a bounded domain in $\mathbb{P}$ and $f=u_{0}+\hat{i} f_{0}$ be hyperholomorphic on $\bar{\Omega}$. Then, for every $z=x_{0}+\hat{i} z_{0} \in \Omega, f$ can be 
expressed as

$$
f(z)=\frac{1}{2 \pi^{2}} \int_{\Omega}-\frac{(\bar{\zeta}-\bar{z})}{|\zeta-z|^{4}} d \omega_{\zeta} f(\zeta)-\frac{1}{2 \pi^{2}} \int_{b \Omega}-\frac{(\bar{\zeta}-\bar{z})}{|\zeta-z|^{4}} d \omega_{\zeta} f(\zeta),
$$

where $\zeta=y_{0}+\hat{i} \zeta_{0}$ and $\omega_{\zeta}=d \zeta_{0} \wedge d \overline{\zeta_{0}}-\hat{i} d y_{0} \wedge d \zeta_{0}$.

Proof. In order to conveniently find the formula of Theorem 3, we put $\phi(\zeta, z)=(\bar{\zeta}-\bar{z})$ and $\psi(\zeta, z)=|\zeta-z|^{4}$. Let $R$ be the distance between $b \Omega$ and $z$. Let $B=B(z, \rho)$ be the open ball of radius $\rho$ with center $z \in \Omega$, where $0<\rho<R$. Suppose $\Omega(z, \rho)=\Omega-B$. Since $\frac{\phi(\zeta, z)}{\psi(\zeta, z)}$ is hyperholomorphic, by the Stokes theorem, we infer

$$
\begin{aligned}
\int_{\Omega(z, \rho)}-\frac{\phi(\zeta, z)}{\psi(\zeta, z)} d \omega_{\zeta} f(\zeta) & =\int_{\Omega(z, \rho)} d\left\{-\frac{\phi(\zeta, z)}{\psi(\zeta, z)} \omega_{\zeta} f(\zeta)\right\}= \\
& =\int_{b \Omega(z, \rho)}-\frac{\phi(\zeta, z)}{\psi(\zeta, z)} \omega_{\zeta} f(\zeta)= \\
& =\int_{b \Omega}-\frac{\phi(\zeta, z)}{\psi(\zeta, z)} \omega_{\zeta} f(\zeta)-\int_{b B}-\frac{\phi(\zeta, z)}{\psi(\zeta, z)} \omega_{\zeta} f(\zeta) .
\end{aligned}
$$

Moreover, we obtain

$$
\begin{aligned}
\int_{b B}-\frac{\phi(\zeta z)}{\psi(\zeta, z)} \omega_{\zeta} f(\zeta) & =\int_{B} d\left(-\frac{\phi(\zeta, z)}{\psi(\zeta, z)} \omega_{\zeta} f(\zeta)\right)= \\
& =\int_{B} d\left\{-\frac{\phi(\zeta, z)}{\psi(\zeta, z)}\right\} \omega_{\zeta} f(\zeta)+\int_{B}\left\{-\frac{\phi(\zeta, z)}{\psi(\zeta, z)}\right\} d \omega_{\zeta} f(\zeta)= \\
& =\frac{1}{\rho^{4}}\left\{\int_{B} f(\zeta) d x_{0} \wedge d z_{0} \wedge d \bar{z}_{0}-\int_{B} \phi(\zeta, z) d \omega_{\zeta} f(\zeta)\right\} .
\end{aligned}
$$

Since $f$ is hyperholomorphic in $\bar{\Omega}$, we have

$$
\begin{gathered}
\lim _{\rho \rightarrow 0} \frac{1}{\rho^{4}} \int_{B} f(\zeta) d x_{0} \wedge d z_{0} \wedge d \bar{z}_{0}=-2 \pi^{2} f(z), \\
\lim _{\rho \rightarrow 0} \frac{1}{\rho^{4}} \int_{B} \phi(\zeta, z) d \omega_{\zeta} f(\zeta)=0 .
\end{gathered}
$$


Hence,

$$
\int_{\Omega}-\frac{\phi(\zeta, z)}{\psi(\zeta, z)} d \omega_{\zeta} f(\zeta)=\int_{b \Omega}-\frac{\phi(\zeta, z)}{\psi(\zeta, z)} \omega_{\zeta} f(\zeta)+2 \pi^{2} f(z) .
$$

Thus, the function $f(z)$ can be expressed as

$$
f(z)=\frac{1}{2 \pi^{2}} \int_{\Omega}-\frac{\phi(\zeta, z)}{\psi(\zeta, z)} d \omega_{\zeta} f(\zeta)-\frac{1}{2 \pi^{2}} \int_{b \Omega}-\frac{\phi(\zeta, z)}{\psi(\zeta, z)} \omega_{\zeta} f(\zeta) .
$$

Corollary 1. Let $\Omega$ be a bounded domain in $\mathbb{T}$ and $f=u_{0}+\hat{i} f_{0}$ be hyperholomorphic in a bounded domain $\Omega \subset \mathbb{T}$. Then, for every $z \in \Omega$, the function $f$ can be expressed as

$$
\begin{aligned}
f(z) & =\frac{1}{2 \pi^{2}} \int_{b \Omega} \frac{(\bar{\zeta}-\bar{z})}{|\zeta-z|^{4}} \omega_{\zeta} f(\zeta)= \\
& =\frac{1}{2 \pi^{2}} \int_{b \Omega} \frac{\left(y_{0}-x_{0}\right)-\hat{i}\left(\zeta_{0}-z_{0}\right)}{\left(\left|y_{0}-x_{0}\right|^{2}+\left|\zeta_{0}-z_{0}\right|^{2}\right)^{2}} \omega_{\zeta} f(\zeta) .
\end{aligned}
$$

Proof. Since $f$ is a hyperholomorphic function on $\Omega$, we have

$$
d \omega_{\zeta} f(\zeta)=0
$$

and the corollary follows by Theorem 3 .

Acknowledgment. The author was supported by the Dongguk University Research Fund 2018 and the National Research Foundation of Korea (NRF) (2017R1C1B5073944).

\section{References}

[1] Deavours C. A. The quaternion calculus. Amer. Math. Monthly, 1973, vol. 80, pp. $995-1008$.

DOI: https://doi.org/10.1080/00029890.1973.11993432.

[2] Fueter R. Über die analytische Darstellung der regulären Funktionen einer Quaternionen-variablen. Comment. Math. Helv., 1935, vol. 8, pp. 371-378. DOI: https://doi.org/10.1007/bf01199562. 
[3] Gürlebeck K., Sprößig W. Quaternionic Analysis and Elliptic Boundary Value Problems. Birkhäuser, Basel, 1989.

DOI: https://doi.org/10.1007/978-3-0348-7295-9.

[4] Gürlebeck K., Sprößig W. Quaternionic and Clifford Calculus for Physicists and Engineers. Wiley, Chichester, 1997.

DOI: https://doi.org/10.1016/s0898-1221(97)90271-6.

[5] Hamilton W. R. Elements of Quaternions. Longmans Green and Company, London, 1866.

DOI: https://doi.org/10.1017/cbo9780511707162.

[6] Kajiwara J., Li X. D., Shon K. H. Regeneration in complex, quaternion and Clifford analysis. Finite or Infinite Dimensional Complex Analysis and Applications, Springer, Boston, 2004, pp. 287-298 .

[7] Kim J. E., Lim S. J., Shon K. H. Regularity of functions on the reduced quaternion field in Clifford analysis. Abstr. Appl. Anal., Volume 2014, Article ID 654798,8 pages.

DOI: https://doi.org/10.1155/2014/654798.

[8] Kim J. E., Lim S. J., Shon K. H. Regular functions with values in ternary number system on the complex Clifford analysis. Abstr. Appl. Anal., Volume 2013, Article ID 136120, 7 pages.

DOI: https://doi.org/10.1155/2013/136120.

[9] Leutwiler H. Modified quaternionic analysis in $\mathbb{R}^{3}$, Complex var. 20 (1992), pp. $19-51$.

DOI: https://doi.org/10.1080/17476939208814584.

[10] Naser M. Hyperholomorphic functions. Siberian Math. J., 1971, vol. 12, pp. $959-968$.

DOI: https://doi.org/10.1007/bf00966540.

[11] Nôno K. Hyperholomorphic functions of a Quaternion variable. Bull. Fukuoka Univ. Edu., 1983, vol. 32, pp. 21-37.

[12] Sudbery A. Quaternionic analysis. Math. Proc. Camb. Phil. Soc., 1979, vol. 85, pp. $199-225$.

DOI: https://doi.org/10.1017/S0305004100055638.

Received May 27, 2019.

In revised form, December 15, 2019.

Accepted December 18, 2019.

Published online January 10, 2020.

Department of Mathematics, Dongguk University,

Gyeongju-si 38066, Republic of Korea

E-mail: jeunkim@pusan.ac.kr 